OPEN ACCESS

Edited by:

Michele Tepedino,

University of L'Aquila, Italy

Reviewed by:

Edoardo Staderini,

Agostino Gemelli University Polyclinic (IRCCS), Italy

Bernardo Souki,

Pontifical Catholic University of Minas

Gerais, Brazil

*Correspondence:

Francesca Gazzani

francescagazzani@hotmail.it

Specialty section:

This article was submitted to Biomaterials,

a section of the journal

Frontiers in Materials

Received: 04 October 2021 Accepted: 09 December 2021

Published: 04 February 2022

Citation:

Gazzani F, Bellisario D, Quadrini F, Parrinello F, Pavoni $C$, Cozza $P$ and Lione $R$ (2022) Comparison Between Different Composite Resins Used for Clear Aligner Attachments: An InVitro Study.

Front. Mater. 8:789143. doi: 10.3389/fmats.2021.789143

\section{Comparison Between Different Composite Resins Used for Clear Aligner Attachments: An In-Vitro Study}

\author{
Francesca Gazzani ${ }^{1 *}$, Denise Bellisario ${ }^{2}$, Fabrizio Quadrini ${ }^{2}$, Francesca Parrinello ${ }^{1}$, \\ Chiara Pavoni ${ }^{1,3}$, Paola Cozza ${ }^{1,3,4}$ and Roberta Lione ${ }^{1,3,4}$
}

${ }^{1}$ Department of Systems Medicine, University of Rome "Tor Vergata", Rome, Italy, ${ }^{2}$ Department of Industrial Engineering, University of Rome "Tor Vergata", Rome, Italy, ${ }^{3}$ Department of Dentistry, UNSBC, Tirana, Albania, "Departmental Faculty of Medicine and Surgery, University of Rome "Unicamillus", Rome, Italy

Attachments are specific features of clear aligner treatment designed to ensure the aligner's retention and the predictability of tooth movements. The properties of composite resin used for their reproduction play a relevant role to preserve their integrity and shape over the time. Thus, the aim of the present evaluation was to compare the mechanical properties and the wear performance of two nanocomposite by means of mechanical and tribological tests. Twelve samples for both flowable nanocomposite (FNC) and conventional nanocomposite (CNC) were created. The two nanocomposites differ in terms of filler volume and viscosity of the mixture. The following tests were performed: thermal analysis and burning test; flat instrumented indentation test and a compression stress relaxation test; tribological analysis. Wear evaluation was performed by means of a contact probe surface profiler and a TayMap software for the $3 \mathrm{D}$ analysis. A customized step-sliding test was conducted to simulate the clinical application of materials with a polymethyl methacrylate (PMMA) ball used as counterpart. Wear evaluation of both resin surfaces and PMMA ball was performed. No differences were found in terms of polymeric nature and quantity of nanoparticles in the matrix. FNC showed lower density values $\left(1.62 \mathrm{~g} / \mathrm{cm}^{3} \pm 0.02\right)$ and inorganic percentage residue $(41 \%)$ than the $\mathrm{CNC}$ (respectively $1.95 \mathrm{~g} / \mathrm{cm}^{3} \pm 0.01$ and $23 \%$ ). Significant differences in terms of decrement of stress values, elastic modulus $(1,114.12 \pm 91.39 \mathrm{MPa})$, and stress relaxation rate $(24.39 \% \pm 3.23)$ were observed for the $\mathrm{CNC}$ when compared to the FNC (respectively, $835.04 \pm 184.73 \mathrm{MPa}$ and $40.19 \% \pm 4.65)$. FNC showed higher values of dynamic friction coefficient $(0.72 \pm 0.017)$ and more worn and deeper profiles than the conventional ones. The step-sliding test with a PMMA ball confirmed a higher friction coefficient for FNC and a greater wear of the PMMA surfaces when used against flowable samples. Lower viscosity of FNC ensures a better adaptation during clinical attachment fabrication, whereas it has a negative impact on mechanical properties. CNC showed greater performance and resistance under mechanical stresses than the flowable ones, resulting in being more suitable for clinical needs.

Keywords: nanocomposite resins, attachments, clear aligner treatment, tribological analysis, mechanical properties 


\section{INTRODUCTION}

Clear aligners treatment (CAT) requires the placement of resin buttons on tooth surfaces to enhance the aligners retention and to create pushing surfaces for a more predictable tooth movement (Morton et al., 2017; D'Anto et al., 2019). In fact, the addition of these auxiliaries, usually referred to as attachments, maximize the contact points and the interaction between the aligner and tooth surfaces. Attachments' position and configuration play a crucial role during the orthodontic treatment since they are strongly related to the force system induced by the aligner. Ideal composite resins suitable for attachment creation need specific esthetic and mechanical properties (Barreda et al., 2017). As for the esthetic aspect, composite resin should be resistant to stain and with similar translucency of the underlying tooth (Feinberg et al., 2016; Barreda et al., 2017). On the other hand, more clinically significant are the mechanical features required. Since they represent needful auxiliary elements for aligners system, their integrity and shapes need to be maintained during the treatment to ensure the functional validity and to not compromise movements' efficiency and aligner fitting (Kravitz et al., 2008; Das et al., 2015; D’Anto et al., 2019; Mantovani et al., 2019). These materials (resin-based dental composite) are widely used in dentistry for dental restorations and orthodontic devices (Aminoroaya et al., 2021). They are usually composed of two phases: an organic resin matrix and an inorganic/organic filler. The organic resin matrix is composed of monomers and lightsensitive initiators, whereas the filler phase consists of different size particles (micro/nano-sized fillers) which determine the material's properties (Fronza et al., 2015; Taheri et al., 2015; Cho et al., 2020; Aminoroaya et al., 2021). The predominant bases monomer used are normally bis-GMA that is sometimes mixed with other dimethacrylates (Ferracane. 2011). Most of the composites contain an activator/initiator system to promote light-activated polymerization of the organic matrix forming cross-linked polymer networks (Nikolaidis. et al., 2019). The most variable contents are represented by the filler particles and their size (Satterthwaite et al., 2009; Satterthwaite et al., 2012). The "nanofill" composites include nanoscale particles characterized by a size range of 1-100 (Ferracane. 2011). Particles' size may range from $20 \mathrm{~nm}$ to $5 \mu \mathrm{m}$ and filler phase overall can represent $70 \%$ of the volume (Lang et al., 1992; Barreda et al., 2017). Attachments' creation usually requires conventional (CNC) or flowable (FNC) dental nanocomposite resins (Ferracane 2011; Mantovani et al., 2019) which mainly differ for filler volume and viscosity. Composite viscosity is determined by monomer composition and filler content. CNC is composed of small particle sizes with a high filler volume and high viscosity of the mixture. On the other hand, FNC present the same small particle sizes of conventional composites with a reduced filler volume, increased resin content, and lower viscosity of the mixture (Bayne et al., 1998; Benetti et al., 2015). This composition produces a composite with an easy adaptation to the attachments template, but a negative impact to wear performance and stability (Clelland et al., 2005). In literature, many studies (Feinberg et al., 2016; Barreda et al., 2017; D’Anto et al., 2019; Mantovani et al., 2019) investigated

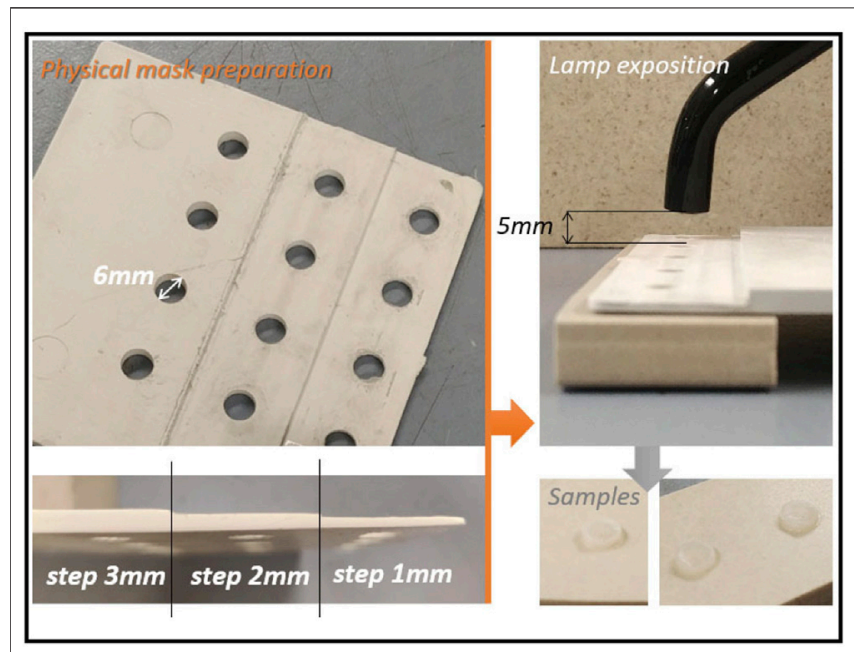

FIGURE 1 | Samples' preparation process: physical mask preparation for sample deposition, lamp exposition, and final samples.

composite resins for this clinical application. D'Anto et al. (2019) demonstrated that composite viscosity does not have any influences on the shape and volume of attachments. However, no data are available on the comparison of mechanical and wear properties of different composite resins available especially when used for attachment. Since the choice of the best material with ideal properties seems to be relevant to ensure the stability and the efficiency of these auxiliary components of CAT, the aim of this study was to analyze the mechanical properties of two nanocomposite resins (CNC vs FNC) with different viscosities and filler volume by means of mechanical and tribological tests.

\section{MATERIALS AND METHODS}

A series of 12 samples (6 FNC, $6 \mathrm{CNC}$ ) were realized for the experimental analysis. Photocurable thickness limits of about $1 \mathrm{~mm}$ for each type of composite were respected and photomasks with three thickness steps have been manufactured to optimize the deposition and the curing phase. All the masks were machined into a polycarbonate sheet in which five circular seats of $6 \mathrm{~mm}$ of diameter have been obtained (Figure 1). The diameter of $6 \mathrm{~mm}$ has been chosen in order to carry out macroscopic mechanical tests as indentation tests and tribological tests for which a contact surface in the order of $5-10 \mathrm{~mm}$ is required as a minimum. An incremental addition of $1 \mathrm{~mm}$ of material has been deposited into the mask's seats and then exposed to UV light until reaching $3 \mathrm{~mm}$ of total thickness. For each sample, the UV lamp (TPC led curing light $50 \mathrm{~N}$, United States) with an irradiance of $800 \mathrm{~mW} / \mathrm{cm}^{2}$ was positioned at $5 \mathrm{~mm}$ of distance and the UV curing time step was set at $25 \mathrm{~s}$. At the end of the curing process, the sample sizes and the weight were measured by means of a digital caliper and a precision balance, and these measures were evaluated as the average dimensions and calculated the density values. The primary endpoints of the present investigation were to evaluate the mechanical properties and the wear behaviour of 


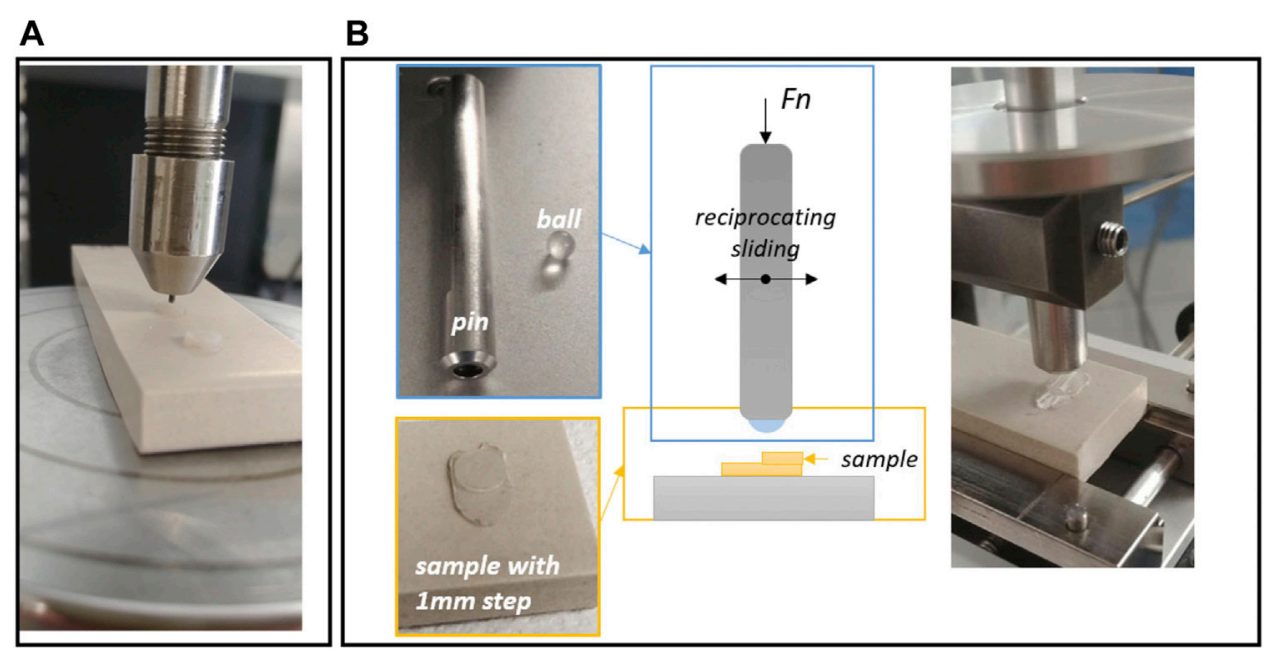

FIGURE 2 | Experimental analysis: (A) Flat indentation test. (B) Step sliding test.

the two nanocomposites. The secondary endpoints were to analyse their thermal properties and to observe the wear behaviour after the simulation of clinical conditions of use through a step-sliding test in combination with a PMMA.

\section{Thermal Analysis and Burning Test}

Given the polymeric nature of the nanocomposite materials, thermal analysis was conducted by differential scanning calorimetry (DSC 7 by Perkin Elmer) in order to evaluate their thermal properties. After samples curing, a small amount $(20 \mathrm{mg})$ of each nanocomposite material was analysed by DSC scanning (range $20-250^{\circ} \mathrm{C}$, rate $10 \mathrm{C} /$ min). Considering the amount of inorganic content for both materials analysed, a burning test was realized. Small quantities (150 mg) of nanocomposites were inserted at $600^{\circ} \mathrm{C}$ for $60 \mathrm{~min}$ into a muffle and after that time the unburned residue was evaluated to calculate the percentage of inorganic content.

\section{Mechanical Tests}

Evaluation of the mechanical properties were performed through a flat instrumented indentation test (Figure 2A) and a compression stress relaxation test. The first analysis was used to assess local mechanical behaviour of nanocomposite samples, while the second test was performed to evaluate the global mechanical properties and the stress relaxation behaviour. The flat indentation tests were carried out on the flat surface of the cylindrical samples, by using a universal material testing machine (Insight/5 by MTS) equipped with an indenter holder. Flat indenter of tungsten carbide was used with a diameter of $1 \mathrm{~mm}$. The tests were performed on 5 samples for each type of material and the indentation was centered with respect to the sample's diameter. The maximum penetration depth imposed was $0.3 \mathrm{~mm}$ ( $10 \%$ of total thickness to avoid the influence of the substrate), the rate was $0.1 \mathrm{~mm} / \mathrm{min}$, and the pre-load was $1 \mathrm{~N}$. The compression stress relaxation tests were performed by means of a universal testing machine (Alliance/50 by MTS) in compression configuration. Five samples have been compressed up to a maximum of $50 \mathrm{MPa}$ by compression plates and fixing the crosshead position and evaluating the stress decrease for $15 \mathrm{~min}$.

\section{Tribological Tests and Wear Evaluation}

Tribological tests with alternative dry-sliding motion were performed on five samples for each type of nanocomposite by a standard tribometer (Linear Reciprocating Tribometer, C.S.M. Instruments, Peseaux, Switzerland) at about $20^{\circ} \mathrm{C}$ and $40 \% \mathrm{RH}$. Tests were performed at $10 \mathrm{~N}$ load and with a back-and-forth sliding (stroke length $4 \mathrm{~mm}$, frequency $2.5 \mathrm{~Hz}$, duration 10,000 cycles) of the alumina ball (6 mm diameter). Samples' wear was assessed by contact probe surface profiler (TalySurf CLI 2000; Taylor Hobson, Leicester, United Kingdom). The profilometer was used to rebuild the wear patterns using a $5 \mu \mathrm{m}$ lateral resolution. The maximum and mean depth, the area, and the volume involved by the action of the counterpart on the surface of the samples were evaluated by using a TayMap software to calculate and qualitatively analyse the $3 \mathrm{D}$ wear patterns.

\section{Customized Test and Simulation of Clinical Application}

A more targeted test was performed to simulate more specifically the clinical condition of use of materials involved in the investigation. The implementation of materials provides their placement on the tooth in thicknesses of $1 \mathrm{~mm}$ to build a step. A step-sliding test has been developed to simulate the insertion and removal of the appliance and the periodic work of the composites in combination with the material of the aligner. A step of each nanocomposite material was created on a ceramic support and a PMMA (polymethyl methacrylate) ball was used as counterpart during step-sliding tests (Figure 2B). To assess the type of polymer of the commercial aligner, a thermal analysis by DSC (DSC 7 by Perkin Elmer) was performed. The step-sliding test was performed with a contact length of $10 \mathrm{~mm}(5 \mathrm{~mm}$ above the 
TABLE 1 | Measured dimensions and weight of the different fabricated samples.

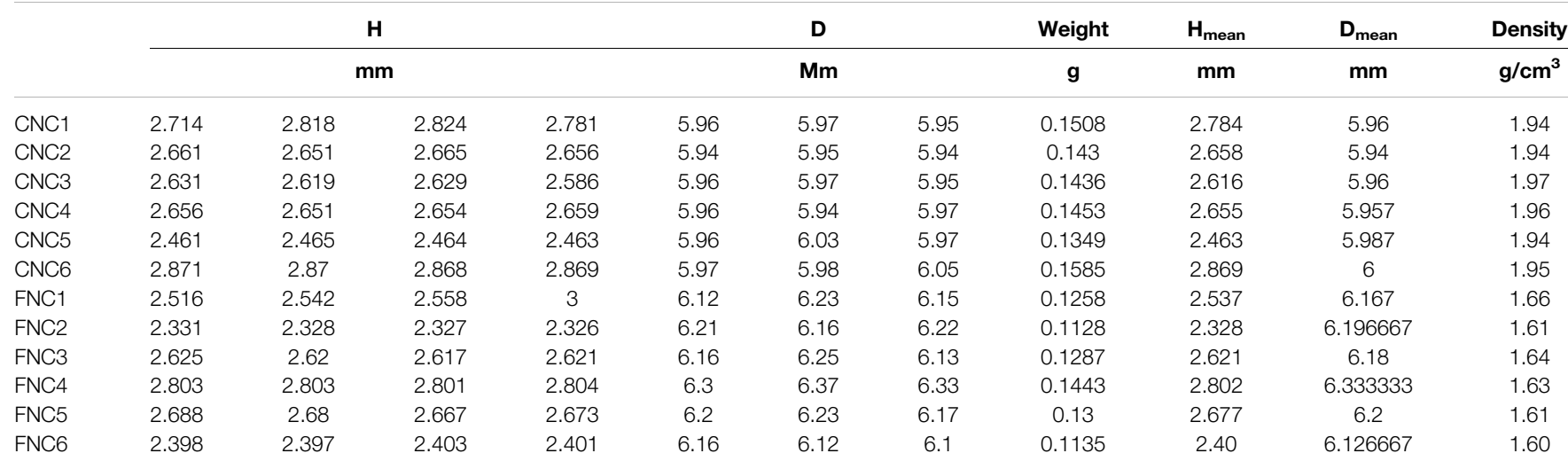

mm, millimeters; g: grams; H: height; D: diameter; FNC, flowable nanocomposite; CNC, conventional nanocomposite.

step and $5 \mathrm{~mm}$ below the step), a duration of 10,000 cycles, a frequency of $2.5 \mathrm{~Hz}$, and a normal load of $1 \mathrm{~N}$. The wear behaviour of the samples was evaluated by contact profilometer (TalySurf CLI 2000; Taylor Hobson, Leicester,
United Kingdom), while the PMMA ball wear was assessed through weight difference before and after the test. The wear values of the nanocomposite samples were also evaluated by $3 \mathrm{D}$ maps of the samples' surface at the top of the step.
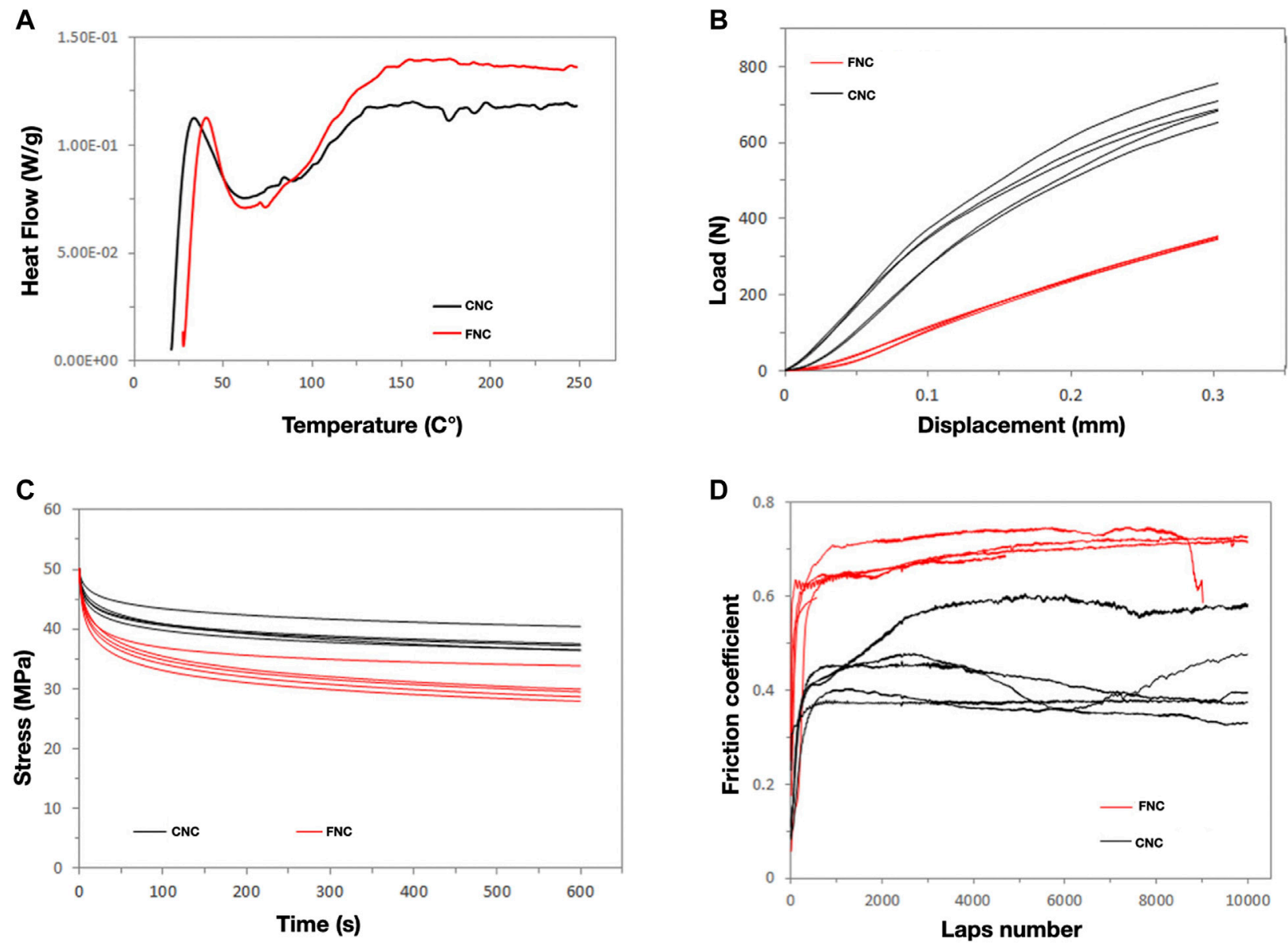

FIGURE 3 | (A) Differential scanning calorimetry curves. (B) Flat indentation curves and trend. (C) Stress relaxation curves. (D) Friction coefficient values monitored during 10,000 laps on the samples' surfaces. The average friction coefficient for each material was evaluated in the range between 6,000 and 8,000 laps at $+1 \mathrm{~mm}$ from the centred position of linear sliding. 

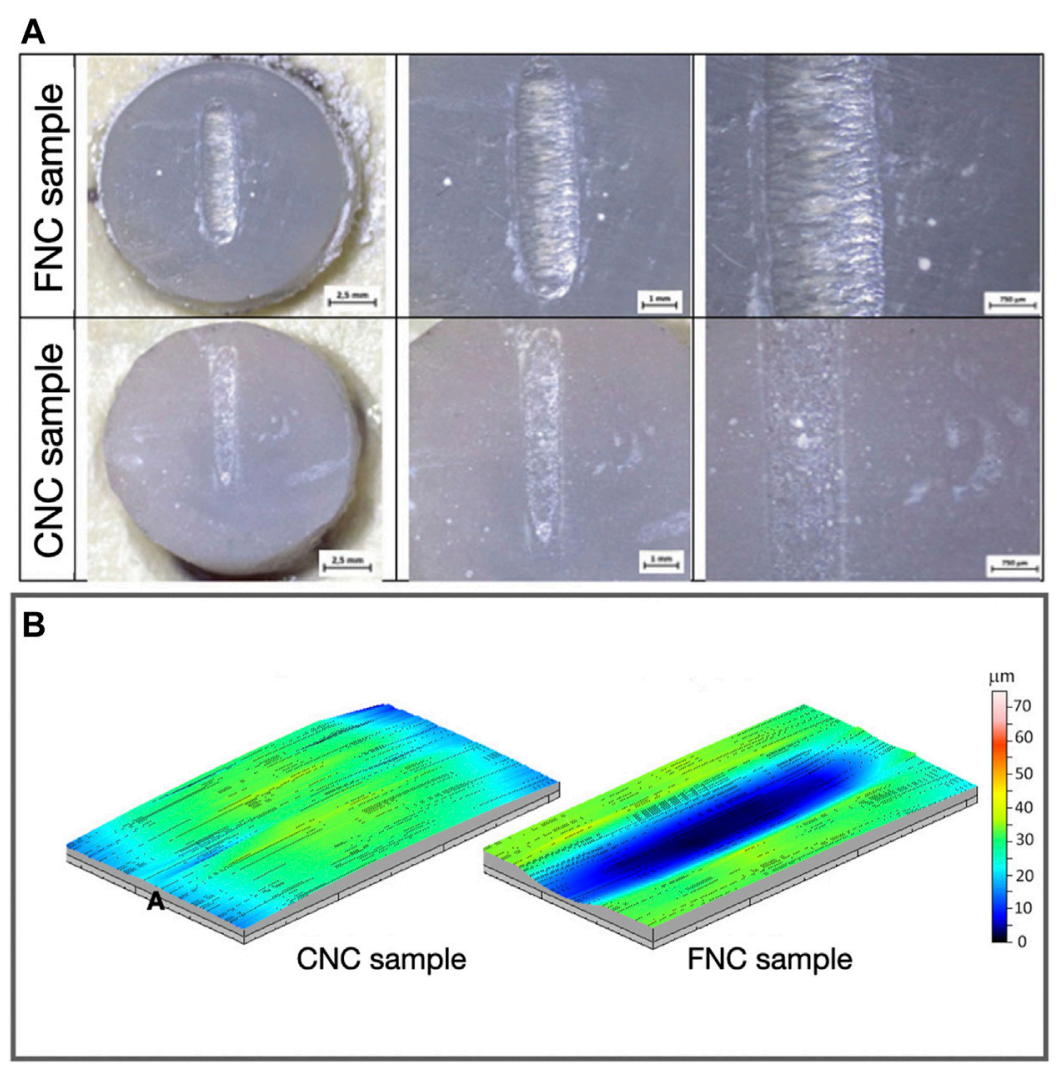

FIGURE 4 | Qualitative evaluation of wear behavior. (A) Sample images after tribological tests. (B) 3D maps of FNC and CNC worn surfaces after tribological tests.

\section{RESULTS}

Table 1 reports the measured dimensions of all fabricated samples in the pilot study and through these values the mean density was evaluated for each type of nanocomposite, in particular $1.62\left(\mathrm{~g} / \mathrm{cm}^{3}\right) \pm 0.02$ and of $1.95\left(\mathrm{~g} / \mathrm{cm}^{3}\right) \pm 0.01$, respectively for FNC and CNC. Analysing the DSC curves for the two materials, no differences were found in terms of polymeric nature of the composites or substantial changes in the quantity of nanoparticles in the matrix. However, the burning test reported an inorganic percentage residue of 41 and $23 \%$, respectively, for the FNC and for CNC (Figure 3A). Form a mechanical point of view, flat indentation tests showed a good repeatability for both materials (Figure 3B). The maximum loads reached at a depth of $0.3 \mathrm{~mm}$ were $364.94 \mathrm{~N} \pm 27.87$ and $723.66 \mathrm{~N} \pm 38.37$, respectively, for flowable and conventional samples. Equally significant differences have been found between the two materials in stress relaxation tests. Specifically, a lower decrease of stress value of the $\mathrm{CNC}$ material was found in comparison to the FNC (Figure 3C). Elastic modulus' evaluations reported greater values observed for the $\mathrm{CNC}$ $(1,114.12 \pm 91.39 \mathrm{MPa})$ than the FNC $(835.04 \pm 184.73 \mathrm{MPa})$. Higher values of stress relaxation were found for the FNC $(40.19 \pm 4.65)$ when compared with CNC $(24.93 \pm 3.23)$. As for tribological test, some FNC samples did not complete the number of cycles as the maximum limit of tangential force detected by the instrument was reached. Higher values of dynamic friction coefficient for FNC were found (Figure 3D). The different wear behaviour of the samples has been also confirmed by the images and 3D maps as shown in Figure 4. The comparison of the worn areas showed deeper wear profiles on FNC surfaces than on the conventional ones (Figures 4, $\mathbf{5 A , B}$ ). As reported in Table 2, worn surface and volume values for the FNC samples were higher than the CNC, and also as expected the maximum and mean depth of the wear were greater for FNC. The step-sliding test with a PMMA ball showed a higher average friction coefficient for FNC in Figure 5C. Also, the PMMA surfaces significantly wore out during the test especially when used against FNC sample. The 3D maps of worn surfaces for both nanocomposites are reported in Figure 6 Extracted values and volume of the two samples (Table 3) showed that the extension of the surface involved is comparable among the two samples, while the worn volume is greater for FNC with a corresponding modification of the PMMA ball geometry.

\section{DISCUSSION}

Composite attachments are geometric buttons routinely required for all CAT. These powerful features are essential to control tooth movements and anchorage units, but they also increase aligners' retention (Mantovani et al., 2019). Rossini et al. concluded that all 

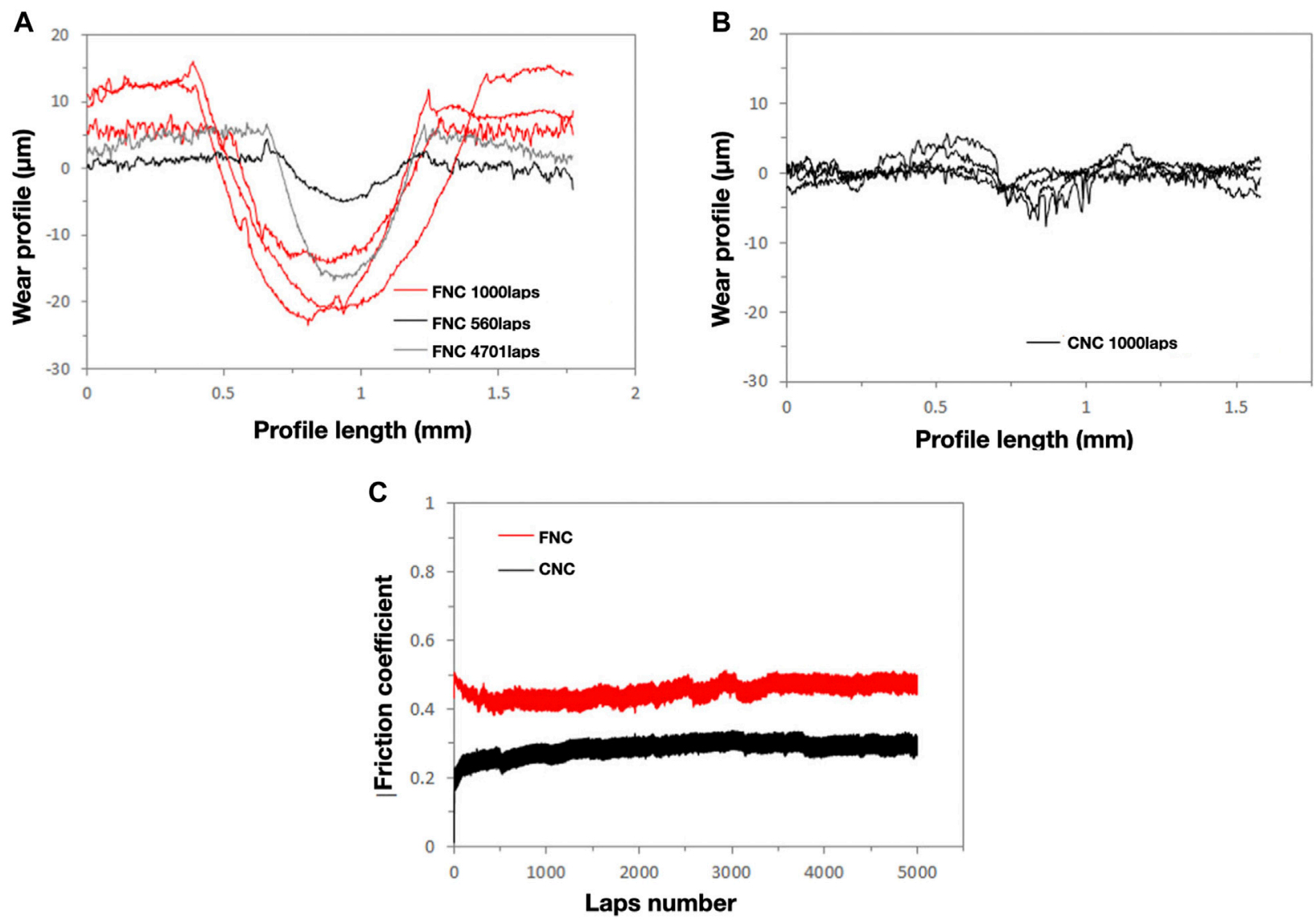

FIGURE 5 | (A) Wear profiles analyses of FNC. (B) Wear profiles analyses of CNC. (C) Friction coefficient trend during step sliding test. The total number of cycles covered was 5,000 , the sliding length was $10 \mathrm{~mm}$. The position at which the friction trends have been evaluated is $+2.5 \mathrm{~mm}$ compared to the central sliding position (on the top of the step).

TABLE 2|Evaluated worn surface and volume, and maximum and mean depth for the worn surfaces.

\begin{tabular}{ccccc}
\hline & Worn surface & Worn volume & Max depth & Mean depth \\
\cline { 2 - 5 } & $\mathbf{m m}^{\mathbf{2}}$ & $\mathbf{m m}^{\mathbf{3}}$ & $\boldsymbol{\mu m}$ & $\boldsymbol{\mu m}$ \\
\hline FNC & $2.158 \pm 0.17$ & $0.030 \pm 0.014$ & $38.933 \pm 15.792$ & $14.867 \pm 3.184$ \\
CNC & $0.901 \pm 0.174$ & $0.002 \pm 0.001$ & $15.951 \pm 5.548$ & $2.193 \pm 0.921$
\end{tabular}

mm, millimeters; $\mu m$, micrometers; FNC, flowable nanocomposite; CNC, conventional nanocomposite.

kinds of attachments had a great impact on the quality and predictability of the tooth movements (Simon et al., 2014; Rossini et al., 2015). For this reason, the selection of composite resins plays a crucial role for the long-term stability of the attachments' shape and for their structural integrity. The aim of the present investigation was to analyse two composite resins with different viscosity and filler volume in order to compare their mechanical properties and to identify which is more suitable for the attachment's reproduction. Our results showed how a greater degree of wear was observed when the attachment was reproduced with the composite presenting a higher percentage of inorganic particles. As a matter of fact, despite the lower content of inorganic filling content, the $\mathrm{CNC}$ resulted denser and it was characterized by a better mechanical resistance (Figure 3A). According to our findings, Barreda et al. (2017) compared attachment surfaces made of two composites with different particle size and filler content by means of Scanning Electron Microscopy (SEM). They concluded that the shape of attachments does not change within 6 months. Thus, the movements related would still be effective in this time interval. Additional data suggested that the low viscosity, defined as the measure of a fluid resistance to flow, is related to a greater elastic modulus indicating that the material offers more resistance to the deformation (Simon et al., 2014). On the other hand, once a strain is given to the FNC material, the viscous component gradually "engages" the deformation (Figure 3C). Both FNC and CNC demonstrated a stress decrease after a strain application as expected by the material's nature; however, the rate of the decrease was higher for CNC. This underlines how for the $\mathrm{CNC}$ the loss of the applied load is faster over time, although the time investigated is relatively short $(15 \mathrm{~min})$. As reported by Tanimoto et al. (2006), elastic modulus is not affected by the filler size, but it depends on stress transmission between the filler and 

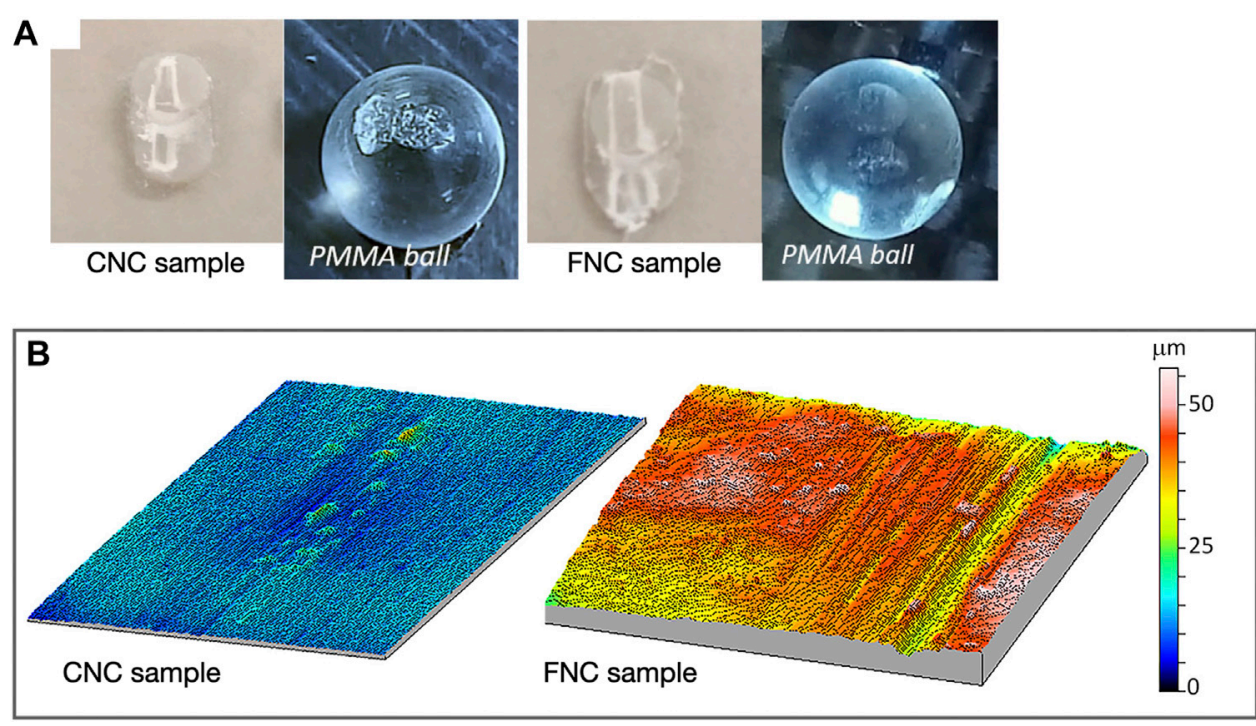

FIGURE 6 | (A) CNC and FNC samples and the respective PMMA balls used after step sliding test. (B) 3D maps of the CNC and FNC samples' worn surface s at the top of the sample step.

TABLE 3 | Worn surface and volume and maximum and mean depth of the worn surface of samples after step sliding test.

\begin{tabular}{lcccc} 
& Worn surface & Worn volume & Max depth & Mean depth \\
\cline { 2 - 5 } & $\mathbf{m m}^{\mathbf{2}}$ & $\mathbf{m m}^{\mathbf{3}}$ & $\boldsymbol{\mu m}$ & $\boldsymbol{\mu m}$ \\
\hline FNC & 1.77 & 0.0038 & 56 & 21.6 \\
CNC & 1.78 & 0.0022 & 7.98 & 1.26 \\
\hline
\end{tabular}

mm, millimeters; $\mu m$, micrometers; FNC, flowable nanocomposite; CNC, conventional nanocomposites.

the matrix. As widely described, resin composites differ from each other in terms of stain resistance, hardness, and wear behaviour (Clelland et al., 2005; Simon et al., 2014; Aminoroaya et al., 2021). In the existing literature (Dasy et al., 2015; Feinberg et al., 2016; Barreda et al., 2017; D’Anto et al., 2019), not many studies analysed the wear behaviour of the composite resins mainly used for attachments reproduction. On the other hand, Barreda et al. (2017) demonstrated that the properties of composites could affect the surface, but not the shape of the attachments during use. More recently, their results have been confirmed by D'Anto et al. (2019) who concluded that viscosity values determine differences in terms of shape and volume. However, the wear behaviour of these materials has not been yet analysed related to their use for attachments reproduction. In this study, the mechanical and wear performances in laboratory tests on the nanocomposites used for the attachments were analysed. In order to carry out such macroscopic evaluations, sample sizes were chosen as small as possible to perform tests under sliding, and as large as possible to have a homogeneous curing under the lamp. Even if the exact clinical conditions are not repeated, at least the single curing step was correctly adopted and it was suitable for the purpose of evaluation and comparison of attachment materials. The tribological test conducted revealed higher values of dynamic friction for the FNC $(0.72 \pm 0.017)$ when compared with the $\mathrm{CNC}(0.41 \pm 0.092)$ with a consequent greater susceptibility to the surface damages (Figure 3D). The evaluation of these damages from a morphological point of view were made by $3 \mathrm{D}$ maps acquisition by a contact profilometer (Figures 4,5 ). In particular, it observed a superficial removal of composite material (worn areas). The qualitative evaluation highlighted deeper grooves and more significant wear traces on FNC samples. The same characteristics have been further confirmed by the step sliding test performed between the nanocomposites and the PMMA ball (Table 3). Also in this case, the results obtained revealed significant differences between the two materials, as shown in Figures 5C, 6. A greater wear has been observed when the PMMA ball was used counterpart in the test with FNC samples indicating a larger abrasive damage among the two materials. As for the samples' surfaces, the 3D analysis (Figure 6) showed that the extension of the surface involved is comparable among the two samples, while the worn volume results were once again greater for the FNC resin.

\section{STRENGTH AND LIMITATIONS}

A strength of the present investigation consisted of having identified the different mechanical and wear properties of the two nanocomposites highlighting the best application of the CNC for attachments reproduction. Wear properties and strength observed during the experimental analysis provide better performance and fitting of aligners, making these materials the best choice for the features design. A limitation of the present study is that although the tests conducted were highly repeatable, they move away from the real conditions of use. Further investigation should be thought to simulate and reproduce the 
insertion and removal of clear aligners and the periodic work of the composites in combination with their material.

\section{CONCLUSION}

CNC resins demonstrated a better mechanical behaviour from a materialistic point of view, and for this reason they seem to be considered the best choice for attachments creation during CAT. Moreover, wear properties in dry conditions and strength observed during the experimental analysis provide better performance and fitting of aligners, making these materials the best choice for the features design.

\section{REFERENCES}

Aminoroaya, A., Neisiany, R. E., Khorasani, S. N., Panahi, P., Das, O., Madry, H., et al. (2021). A Review of Dental Composites: Challenges, Chemistry Aspects, Filler Influences, and Future Insights. Composites B: Eng. 216, 108852. doi:10.1016/j.compositesb.2021.108852

Barreda, G. J., Dzierewianko, E. A., Muñoz, K. A., and Piccoli, G. I. (2017). Surface Wear of Resin Composites Used for Invisalign(R) Attachments. Acta Odontol. Latinoam. 30, 90-95.

Bayne, S. C., Thompson, J. Y., Swift, E. J., Jr, Stamatiades, P., and Wilkerson, M. (1998). A Characterization of First-Generation Flowable Composites. J. Am. Dental Assoc. 129 (5), 567-577. doi:10.14219/jada.archive.1998.0274

Benetti, A., Havndrup-Pedersen, C., Honoré, D., Pedersen, M., and Pallesen, U. (2015). Bulk-fill Resin Composites: Polymerization Contraction, Depth of Cure, and gap Formation. Oper. Dent 40, 190-200. doi:10.2341/13-324-1

Cho, K., Sul, J.-H., Stenzel, M. H., Farrar, P., and Prusty, B. G. (2020). Experimental Cum Computational Investigation on Interfacial and Mechanical Behavior of Short Glass Fiber Reinforced Dental Composites. Composites Part B: Eng. 200, 108294. doi:10.1016/j.compositesb.2020.108294

Clelland, N. L., Pagnotto, M. P., Kerby, R. E., and Seghi, R. R. (2005). Relative Wear of Flowable and Highly Filled Composite. The J. Prosthetic Dentistry 93, 153-157. doi:10.1016/j.prosdent.2004.11.006

D’Antò, V., Muraglie, S., Castellano, B., Candida, E., Sfondrini, M. F., Scribante, A., et al. (2019). Influence of Dental Composite Viscosity in Attachment Reproduction: An Experimental In Vitro Study. Materials (Basel) 12 (23), 4001. doi:10.3390/ma12234001

Dasy, H., Dasy, A., Asatrian, G., Rózsa, N., Lee, H.-F., and Kwak, J. H. (2015). Effects of Variable Attachment Shapes and Aligner Material on Aligner Retention. Angle Orthod. 85, 934-940. doi:10.2319/091014-637.1

Feinberg, K. B., Souccar, N. M., Kau, C. H., Oster, R. A., and Lawson, N. C. (2016). Translucency, Stain Resistance, and Hardness of Composites Used for Invisalign Attachments. J. Clin. Orthod. 50, 170-176.

Ferracane, J. L. (2011). Resin Composite-State of the Art. Dental Mater. 27 (1), 29-38. doi:10.1016/j.dental.2010.10.020

Fronza, B. M., Rueggeberg, F. A., Braga, R. R., Mogilevych, B., Soares, L. E. S., Martin, A. A., et al. (2015). Monomer Conversion, Microhardness, Internal Marginal Adaptation, and Shrinkage Stress of Bulk-Fill Resin Composites. Dental Mater. 31 (12), 1542-1551. doi:10.1016/j.dental.2015.10.001

Kravitz, N. D., Kusnoto, B., Agran, B., and Viana, G. (2008). Influence of Attachments and Interproximal Reduction on the Accuracy of Canine Rotation with Invisalign. A Prospective Clinical Study. Angle Orthod. 78, 682-687. doi:10.2319/0003-3219(2008)078[0682:IOAAIR]2.0.CO;2

Lang, B. R., Jaarda, M., and Wang, R.-F. (1992). Filler Particle Size and Composite Resin Classification Systems. J. Oral Rehabil. 19, 569-584. doi:10.1111/j.13652842.1992.tb01487.x

Mantovani, E., Castroflorio, E., Rossini, G., Garino, F., Cugliari, G., Deregibus, A., et al. (2019). Scanning Electron Microscopy Analysis of Aligner Fitting on anchorage Attachments. J. Orofac. Orthop. 80, 79-87. doi:10.1007/s00056-01800167-1

\section{DATA AVAILABILITY STATEMENT}

The original contributions presented in the study are included in the article/Supplementary Material, further inquiries can be directed to the corresponding author.

\section{AUTHOR CONTRIBUTIONS}

PC, RL, and FG proposed the research idea and wrote the article. DB and FQ improved the idea and performed the experimental analysis. They also contributed to the writing of the paper. CP and FP assisted the experimental analysis. All authors reviewed the article.

Morton, J., Derakhshan, M., Kaza, S., and Li, C. (2017). Design of the Invisalign System Performance. Semin. Orthod. 23, 3-11. doi:10.1053/j.sodo.2016.10.001

Nikolaidis, A. K., Koulaouzidou, E. A., Gogos, C., and Achilias, D. S. (2019). Synthesis and Characterization of Dental Nanocomposite Resins Filled with Different Clay Nanoparticles. Polymers 11 (4), 730. doi:10.3390/ polym 11040730

Rossini, G., Parrini, S., Castroflorio, T., Deregibus, A., and Debernardi, C. L. (2015). Efficacy of clear Aligners in Controlling Orthodontic Tooth Movement: A Systematic Review. Angle Orthod. 85, 881-889. doi:10.2319/061614-436.1

Satterthwaite, J. D., Maisuria, A., Vogel, K., and Watts, D. C. (2012). Effect of Resin-Composite Filler Particle Size and Shape on Shrinkage-Stress. Dental Mater. 28, 609-614. doi:10.1016/j.dental.2012.01.007

Satterthwaite, J. D., Vogel, K., and Watts, D. C. (2009). Effect of Resin-Composite Filler Particle Size and Shape on Shrinkage-Strain. Dental Mater. 25, 1612-1615. doi:10.1016/j.dental.2009.08.012

Simon, M., Keilig, L., Schwarze, J., Jung, B. A., and Bourauel, C. (2014). Treatment Outcome and Efficacy of an Aligner Technique - Regarding Incisor Torque, Premolar Derotation and Molar Distalization. BMC Oral Health 14, 68. doi:10.1186/1472-6831-14-68

Taheri, M. M., Abdul Kadir, M. R., Shokuhfar, T., Hamlekhan, A., Shirdar, M. R., and Naghizadeh, F. (2015). Fluoridated Hydroxyapatite Nanorods as Novel Fillers for Improving Mechanical Properties of Dental Composite: Synthesis and Application. Mater. Des. 82, 119-125. doi:10.1016/ j.matdes.2015.05.062

Tanimoto, Y., Kitagawa, T., Aida, M., and Nishiyama, N. (2006). Experimental and Computational Approach for Evaluating the Mechanical Characteristics of Dental Composite Resins with Various Filler Sizes. Acta Biomater. 2 (6), 633-639. doi:10.1016/j.actbio.2006.06.006

Conflict of Interest: The authors declare that the research was conducted in the absence of any commercial or financial relationships that could be construed as a potential conflict of interest.

The reviewer BS declared a past collaboration with several of the authors CP, PC, and RL to the handling editor.

Publisher's Note: All claims expressed in this article are solely those of the authors and do not necessarily represent those of their affiliated organizations, or those of the publisher, the editors, and the reviewers. Any product that may be evaluated in this article, or claim that may be made by its manufacturer, is not guaranteed or endorsed by the publisher.

Copyright (c) 2022 Gazzani, Bellisario, Quadrini, Parrinello, Pavoni, Cozza and Lione. This is an open-access article distributed under the terms of the Creative Commons Attribution License (CC BY). The use, distribution or reproduction in other forums is permitted, provided the original author(s) and the copyright owner(s) are credited and that the original publication in this journal is cited, in accordance with accepted academic practice. No use, distribution or reproduction is permitted which does not comply with these terms. 